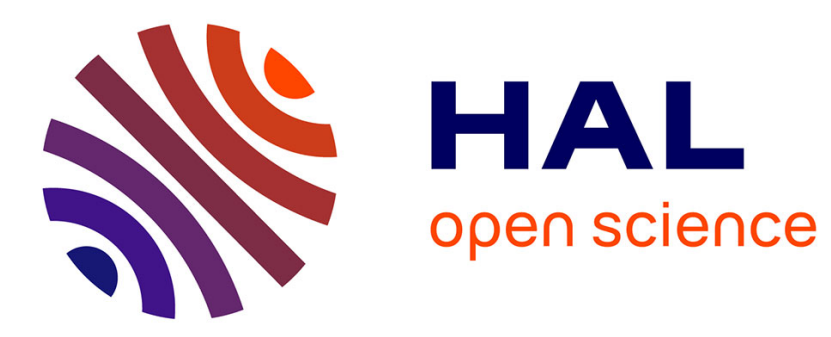

\title{
La moyenne vallée du Rhône, de l'Isère à l'Ardèche
}

Cécile Jung, Thierry Odiot

\section{To cite this version:}

Cécile Jung, Thierry Odiot. La moyenne vallée du Rhône, de l'Isère à l'Ardèche. Gallia - Archéologie de la France antique, 1999, Le Rhône romain, 56, pp.81-90. 10.3406/galia.1999.3245 . hal-01913103

\section{HAL Id: hal-01913103 \\ https://hal.science/hal-01913103}

Submitted on 16 Jan 2020

HAL is a multi-disciplinary open access archive for the deposit and dissemination of scientific research documents, whether they are published or not. The documents may come from teaching and research institutions in France or abroad, or from public or private research centers.
L'archive ouverte pluridisciplinaire HAL, est destinée au dépôt et à la diffusion de documents scientifiques de niveau recherche, publiés ou non, émanant des établissements d'enseignement et de recherche français ou étrangers, des laboratoires publics ou privés.

\section{(1) $\$$}

Distributed under a Creative Commons Attribution - NonCommercial - NoDerivatives| 4.0 


\title{
LA MOYENNE VALLÉE DU RHÔNE, DE L'ISÈRE À L'ARDÈCHE
}

\author{
Cécile Jung et Thierry ODIOT
}

\begin{abstract}
Mots-clés. Tricastin, gués, bacs, barques, navigation, itinéraires terrestres, voie d'Agrippa, diverticules, cours du Rhône, régime fluvial, habitats, centuriation, endiguement, drainage.

Key-words. Tricastin, fords, pontoon bridges, boats, navigation, land roads, via Agrippina, tracks, the Rhône course, river system, settlements, centuriation, dyking, drain.

Résumé. Les récents travaux de recherche sur la vallée du Rhône et son environnement dans l'Antiquité permettent une relecture des sources sur le commerce et l'occupation des sols dans la région. Le Rhône n'apparaît plus comme une frontière physique infranchissable, tant pour les limites politiques des peuples (Ségauvellaunes, Helviens, Cavares) que pour les axes commerciaux. Le franchissement du fleuve par gués, bacs el barques, la remontée de la vallée du Rhône par les itinéraires terrestres, voie d'Agrippa et diverticules, les passages des affluents du Rhône sont abordés. Il faut aussi repenser notre perception du fleuve. L'image actuelle d'un fleuve domestiqué, celle du siècle dernier d'un fleuve sauvage aux crues fréquenles el désastreuses ne sont pas pertinentes pour l'Antiquité. Le cours antique et son régime on tpermis à toule une série d'habitats de s'installer près du lit majeur. Ces installations allaient de pair avec des travaux d'endiguement de drainage souvent liés à des centuriations.
\end{abstract}

\begin{abstract}
Recent research on the Rhône valley and its environment in Antiquily has made possible a new reading of evidence regarding regional trade and settlement pallern. The Rhône is no more considered as a physical impassable border concerning tribal political boundaries (Scgauvellones, Helvii, Cavares) as well as trade axis. Following topics are examined : the river crossing through fords, by pontoonbridges and boats, land routes, via Agrippina and tracks going up the Rhône valley, passages across its affluents. Our understanding of the river needs also thinking out again. The actual picture of a domesticated river and the picture of last century portraying a turbulent river with frequent and appalling over-flows are not relevant dealing with ancient times. The ancient river course and its system have allowed the establishment of series of settlements along the main bed. They went together with dyking and drains often connected to centuriations.
\end{abstract}

Les travaux menés depuis une quinzaine d'années dans la moyenne vallée du Rhône (entre le confluent de l'Isère et celui de l'Ardèche), dans le cadre de programmes collectifs de recherches ( $c f$. Leveau, supra, p. 5), ainsi que les nombreuses interventions en archéologie préventive permettent de dresser le tableau de cet espace géographique tant sur le plan de l'organisation des paysages antiques (réseaux cadastrés, répartition spatiale et dynamique du peuplement, réseau de communications) que sur celui de leur évolution géomorphologique. Ces différents éléments nous permettent de mieux comprendre le fonctionnement de cette partie de la vallée. Dans ce cadre général nous traiterons surtout du secteur du Tricastin qui est particulièrement bien documenté.

\section{LE RHÔNE UNE LIMITE ?}

Le Rhône est actuellement une frontière administrative ancrée sur une réalité physique. Du nord au sud, il sépare les départements de l'Ardèche et du Gard de leurs 
pendants sur la rive gauche : la Drôme et le Vaucluse. En 1867, sur la carte d'état-major, seuls huit ponts le traversent sur $80 \mathrm{~km}$. Toutefois, les données sur les limites préromaines, romaines, médiévales et modernes permettent de relativiser cette image de frontière difficilement franchissable qui est une représentation moderne.

\section{LE PROBLÈME DES SÉGOVELLAUNES}

Ainsi, au nord de la zone étudiée, les limites du territoire des Ségovellaunes définies par G. Barruol (1975) montrent que le fleuve ne constitue probablement pas une frontière politique. Ce peuple préromain serait limité à l'est par le massif du Vercors (où sont situés les Vercoarii), au nord par le cours de l'Isère (marquant la frontière avec les Allobroges), au sud par le cours de la rivière Drôme, et à l'ouest par les premiers contreforts des Cévennes.

Si personne ne s'est penché depuis les dix dernières années sur le problème des frontières nord et est des Ségovellaunes, quelques articles ont abordé le sujet pour les frontières sud et ouest.

Au sud, G. Chouquer et T. Odiot (1984), puis C. Jung (1991) et F. Vaireaux (1993) ont utilisé les limites des réseaux cadastrés de Valence pour restituer les limites du territoire des Ségovellaunes. Ainsi la cité s'étendrait audelà de la Drôme à la hauteur de Cliousclat et Mirmande. Le même argument est utilisé par A. Blanc (1953) pour attribuer à ce peuple les premiers contreforts de la rive ardéchoise à la hauteur de Soyons et Saint-Péray.

Ce type de raisonnement, au premier abord séduisant, semble aujourd'hui peu fondé. En effet, la matérialisation d'un réseau n'est pas synonyme d'une appartenance territoriale, comme le montre l'exemple de l'extension du cadastre B d'Orange sur les territoires des Helviens et des Volques Arécomiques, et bien d'autres exemples.

La présence attestée d'une même limitatio sur les deux rives du fleuve, comme le réseau cadastral de la cité de Valence ou, plus au sud, le réseau B d'Orange, montre combien la vallée était perçue comme un ensemble cohérent, le Rhône n'étant pas considéré comme un obstacle au franchissement est-ouest.

Dans le cas de Valence, l'attribution du site majeur de Soyons (sur la rive ardéchoise) et de toute la région de Saint-Péray aux Ségovellaunes, si l'on ne retient pas les

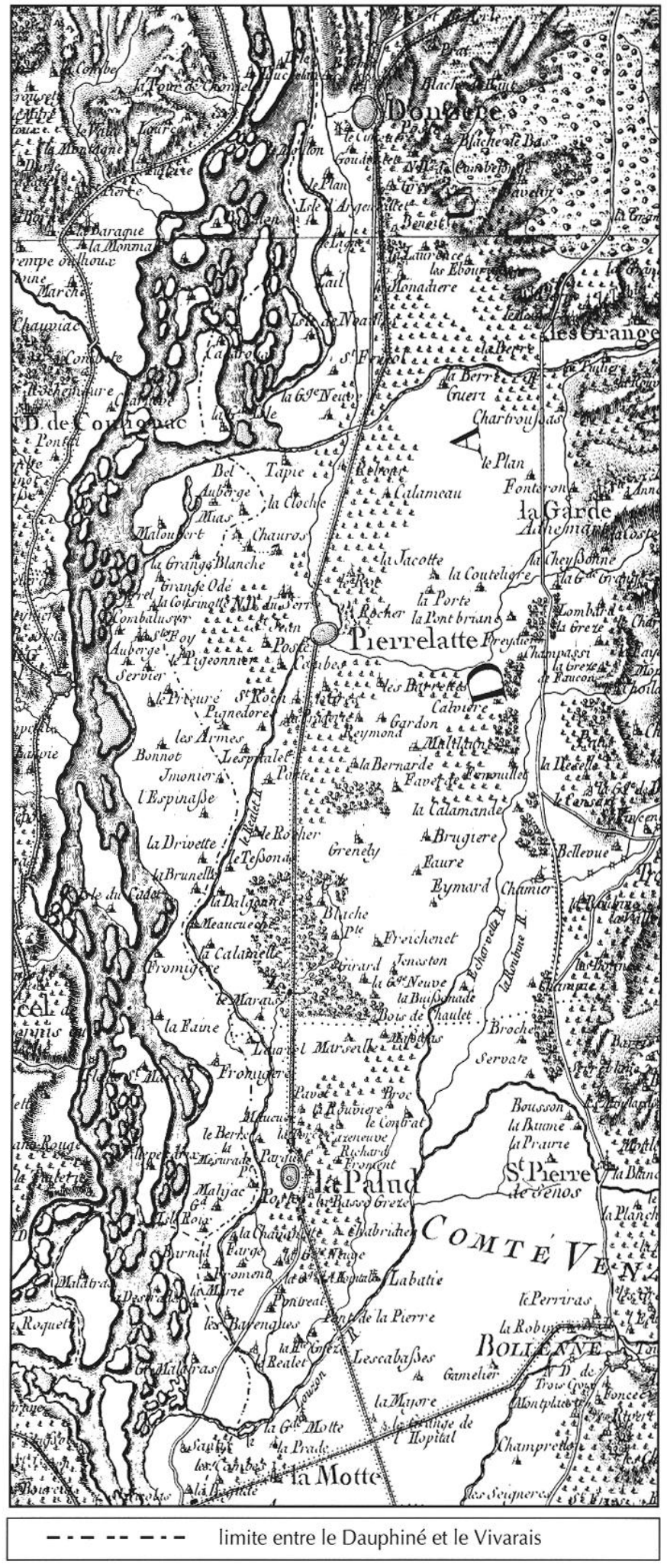

Fig. 32 - Limites des territoires du Vivarais el du Dauphiné sur la carte de Cassini (1772). 
limites physiques de l'extension cadastrale, ne repose que sur l'onomastique. Les découvertes effectuées en face de la cité, en rive droite (commune de Granges-lès-Valence), sans atteindre la densité ni la structuration des occupations de Saint-Romain-en-Gal, plaident pour l'existence probable d'un faubourg de la ville sur la rive ardéchoise.

La présence d'une occupation dense, de part et d'autre du fleuve, montre surtout combien celui-ci ne marquait pas une barrière physiquement ou psychologiquement infranchissable ( $c f$. Béal et Odiot, infra, p. 94-96). Selon toute vraisemblance, l'activité économique n'était en rien entravée par la présence du fleuve. Si l'on place la frontière politique entre les Ségovellaunes et les Helviens à la hauteur de Valence, à l'emplacement du Rhône, il faut envisager qu'un faubourg d'une cité puisse appartenir à un autre territoire, ce qui, bien qu'envisageable, n'a encore jamais été attesté. En l'absence d'éléments plus solides il paraît donc possible d'attribuer aux Ségovellaunes cette partie de l'Ardèche.

\section{LES LIMITES DES ÉVÊCHÉS MÉDIÉVAUX}

Plus au sud, les limites de l'évêché de Viviers renforcent l'impression d'une vallée considérée comme un ensemble cohérent d'une rive à l'autre. Son extension dans la plaine de Pierrelatte, à la hauteur de Bourg-SaintAndéol, est en effet clairement attestée. Pour la fin de la période moderne, la carte de Cassini montre également que le Rhône ne constitue en rien une frontière politique entre le Vivarais et le Dauphiné à la hauteur de Loriol-sur-Drôme et de Pierrelatte (fig. 32).

La perception du Rhône comme frontière politique et administrative dans la moyenne vallée du Rhône apparaît donc comme un usage récent surtout perceptible après la Révolution française. Rien ne permet d'affirmer que les cités protohistoriques et antiques aient considéré le Rhône comme une barrière infranchissable et de nombreuses têtes de pont semblent avoir existé (Barruol, 1975 , p. 270).

\section{FRANCHIR LE RHÔNE}

La déclivité du Rhône, entre Valence et Pont-SaintEsprit, varie entre 0,76 et $0,80 \mathrm{~m} / \mathrm{km}$. Cette pente qui est la plus forte des grands cours d'eaux européens (Saône $0,04 \mathrm{~m} / \mathrm{km}$ de même que le Rhin en aval de Strasbourg, Loire $0,10 \mathrm{~m} / \mathrm{km}$ ) induit un débit torrentiel (Bethemont, 1972, p. 88). Celui-ci est amplifié suivant les saisons par les crues alternées du fleuve et de ses affluents. La fonte des neiges (régime alpin) et les orages méditerranéens (pour le sud de la zone étudiée), qui donnent un caractère dévastateur à ses affluents, sont autant de possibilités de modification du cours. Au printemps, ces facteurs se conjuguent et s'amplifient jusqu'à perturber fortement la navigation (Denel, 1970).

Le lit mineur du Rhône évolue à chaque saison de façon radicale. Ainsi les archives modernes mentionnent de nombreux conflits entre les riverains relatifs à l'appropriation de telle ou telle île ou à la perception de l'impôt sur des terres emportées (Peloux, 1994).

Les données paléoclimatiques et paléohydrologiques pour l'Antiquité restent encore lacunaires mais permettent d'avoir cependant une idée sur le cours du fleuve dans sa moyenne vallée ( $c f$. Provansal et al., supra, p. 21-25).

De la conquête au milieu du $\mathrm{I}^{\mathrm{cr}} \mathrm{s}$., l'ensemble des cours d'eau de la moyenne vallée du Rhône (bassin valdainais et Tricastin) sont en phase d'incision (Berger, 1995, p. 96). La représentation, dans la forma de la centuriation d'Orange B, du cours du Rhône et de ses affluents préalpins (la Berre et le Lez notamment) dans la plaine du Tricastin montre un style fluvial contrasté (fig. 33).

À la hauteur de Donzère, le fleuve après avoir traversé le défilé dessine une série de méandres, tout comme son affluent la Berre. Ce style fluvial est en général accompagné d'un chenal profond, d'un champ d'inondation réduit et correspond à un régime hydrique régulier.

Plus au sud, à la hauteur des confluents avec le Lez et l'Ardèche, le fleuve dessine une série d'îles et les mentions « remisa in aquam " et «nova aera" (Piganiol, 1962 ; Odiot, 1994) montrent combien ce cours était instable en 77 après J.-C. Les documents cadastraux révèlent ainsi une image contrastée du cours du Rhône. On peut s'interroger sur la valeur cartographique de la représentation du fleuve, sur les marbres, mais les observations archéologiques concordantes montrent dès la seconde partie du $\mathrm{I}^{\mathrm{er}} \mathrm{s}$. un exhaussement du lit des affluents du Rhône attesté dans le bassin valdainais et le Tricastin (Berger, 1995, p. 104).

La carte de Cassini, levée vers 1770, apparaît comme un document important pour l'observation du cours du fleuve avant les premiers grands travaux d'endiguement 


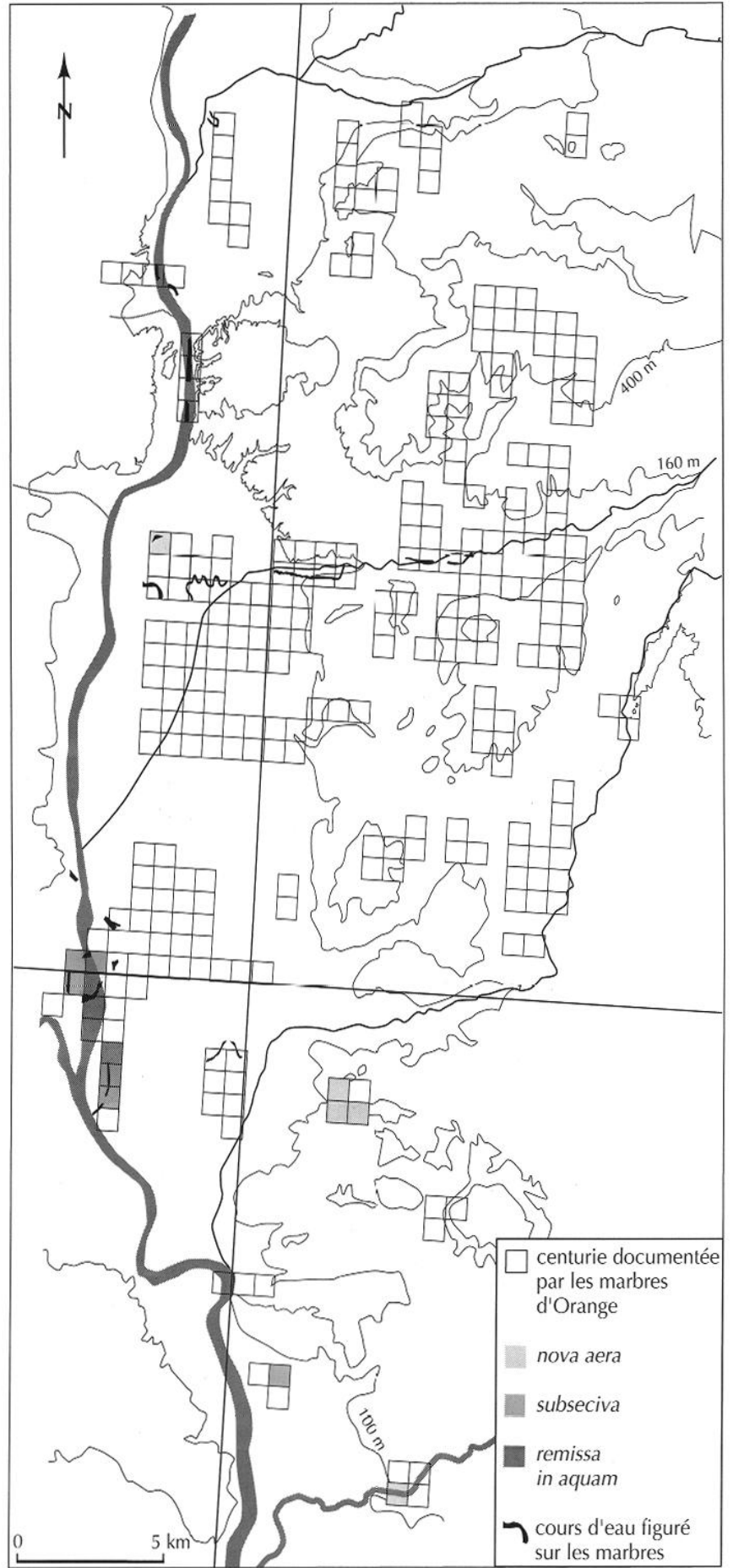

Fig. 33 - Spatialisation des fragments de marbre du cadastre B d'Orange (DAO, C. Jung).

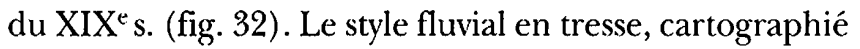
de façon certainement stéréotypée sur cette carte de la fin du XVIII ${ }^{\mathrm{e}}$ s., indique néanmoins un exhaussement durable du lit du Rhône et un élargissement de sa bande d'activité. Le cours du Rhône est alors marqué par une extrême mobilité des îles et des rives, par des zones d'instabilité à l'amont des cônes de déjections des affluents et par des secteurs paraissant plus stables. La mobilité des rives et des îles ainsi que le caractère torrentiel du débit du Rhône sont autant d'obstacles à son franchissement. Il n'en va pas de même durant les périodes d'étiage, où ce style fluvial favorise les franchissements. Cette situation a pu se présenter au cours de l'Antiquité lors des crises hydroclimatiques identifiées à la fin du $\mathrm{I}^{\mathrm{cr}}$ s. et aux $\mathrm{VI}^{e}$-VIII ${ }^{\mathrm{e}}$ s. en moyenne vallée du Rhône ( $c f$. Provansal $e t$ al., supra, p. 13-32).

\section{LES GUÉS}

Dès le Néolithique moyen, les traces d'une circulation est-ouest (échanges, courants culturels transalpins...) apparaissent clairement dans la culture matérielle et, sans minimiser les possibilités de franchissement par embarcation, une série de gués est proposée par A. Beeching (Beeching et al., 1989). Les hauts-fonds localisés dans le lit mineur du fleuve ne correspondent pas forcément aux mêmes formations. On rencontre par exemple des hauts-fonds mobiles constitués par une accumulation de sédiments liée à une rupture de charge, dont la localisation peut varier en fonction des accidents saisonniers. Les affleurements rocheux, par définition stables quelle que soit la physionomie du cours du fleuve, forment eux aussi des hauts-fonds et peuvent également favoriser l'accumulation de sédiments. À la hauteur de Bourg-Saint-Andéol, un ressaut de calcaire urgonien ne laissait que $80 \mathrm{~cm}$ d'eau vive circuler durant l'été 1876 (Poinsart, 1992).

L'ensemble de ces hauts-fonds favorise, en période d'étiage, la traversée du fleuve et correspond probablement à des passages à gué. La localisation des hauts-fonds du Rhône entre Donzère et Pont-Saint-Esprit coïncide très souvent avec celle des chemins allant au Rhône et fonctionnant dès l'Antiquité (Poinsart, 1992 ; Chouquer, Jung, 1996) (fig. 34).

\section{LES BACS ET LES BARQUES}

Durant la période moderne, tant pour la circulation humaine que pour la transhumance, une série de bacs est 


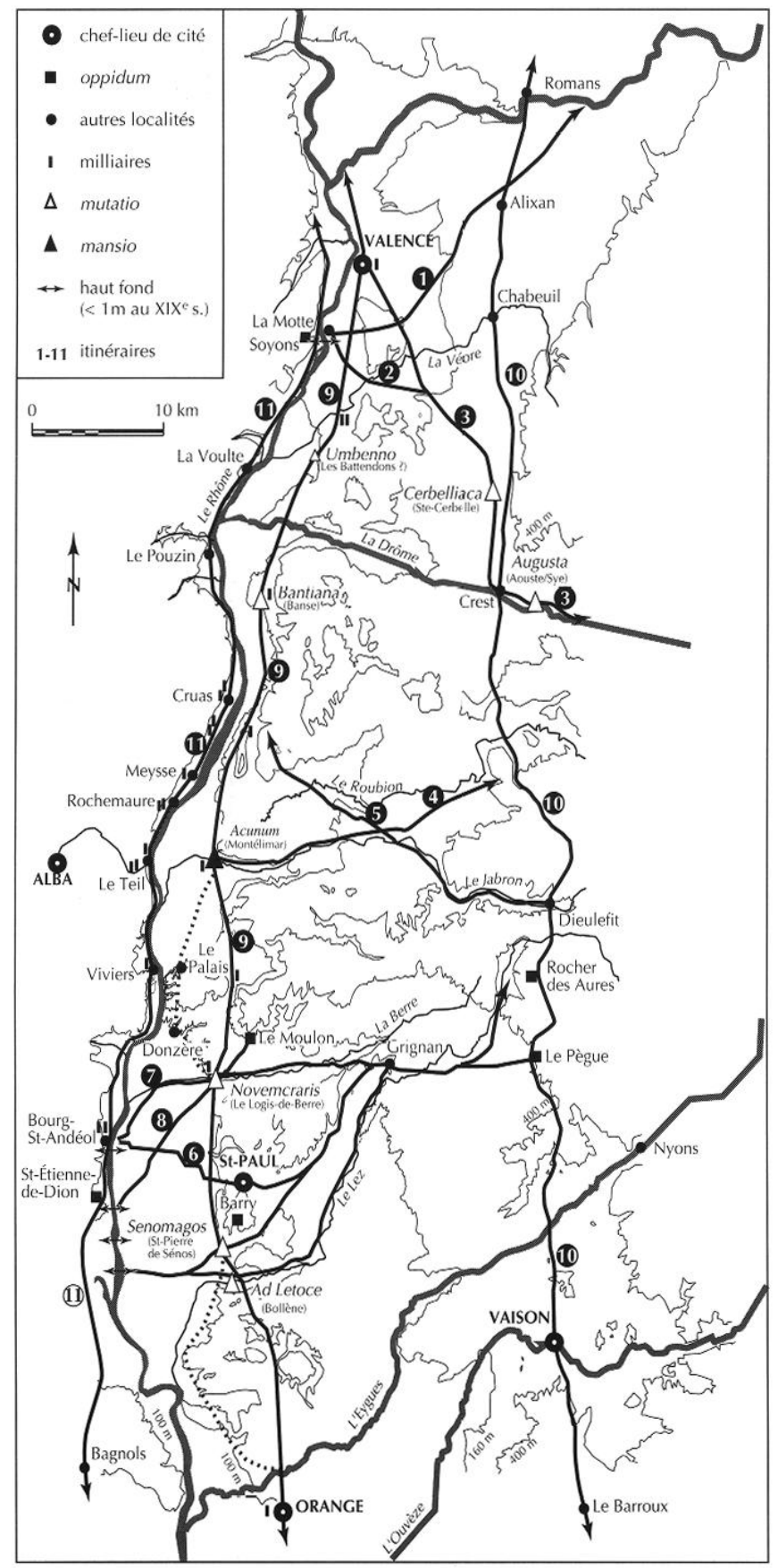

Fig. 34 - Carte des itinéraires terrestres antiques el localisation des passages aisés connus sur le Rhône (DAO, C. Jung).

attestée le long du fleuve (Cogoluhene, 1980). Deux types de bacs ont pu fonctionner durant l'Antiquité, mais aucun embarcadère, structure légère par excellence, n'est assurément attesté par des vestiges archéologiques. Le premier type de bac est celui à rames. Il s'agit d'un radeau dirigé et propulsé par rames ou perches et qui est certainement le moyen de franchissement des cours d'eau le plus ancicn. De pctite capacité mais extrêmement souple dans son utilisation, il s'adapte aux bouleversements du lit du fleuve. Le bac à traille pendulaire nécessite en revanche un point d'ancrage. Il fixe son ancre, à laquelle sont attachés un cordage et le bac, en plein fleuve, si possible sur un haut-fond. La traversée du fleuve s'effectue en utilisant la force du courant. Ce mode de franchissement est envisageable si le lit du cours d'eau est unique et de largeur modérée. Par ailleurs, ils gênent la circulation nord-sud et leur présence induit une batellerie relativement peu abondante.

Ici encore, la présence de hauts-fonds fixes peut induire un franchissement facile du cours du Rhône, ce qui n'empêche en rien la création de bacs pouvant fonctionner durant quelques années dans un secteur plus mobile. Bien évidemment ces structures posent le problème des nautes et des utriculaires ( $c f$. Christol et Fiches, infra, p. 145-146 et 152-155).

Dans la mesure où aucune donnée n'atteste, de façon certaine, la présence d'un pont entre Vienne et Arles durant l'Antiquité, seules la convergence d'une série d'itinéraires routiers est-ouest vers des hauts-fonds (passages aisés du fleuve) et la possibilité d'un passage à bac dans différents secteurs pondèrent donc l'image du Rhône infranchissable. En effet, dans cette zone le seul pont mentionné est celui de Valence. L'existence probable d'un faubourg sur la rive droite et l'intense circulation entre les deux rives rendent nécessaire la présence d'un bac et peut-être d'un gué, mais celle d'un pont reste à démontrer ( $c f$. Béal et Odiot, infra, p. 95-96). Outre Valence, Granges-lès-Valence, d'autre sites « doubles » viennent rythmer les passages sur le fleuve et servir de débarcadères aux circulations nord-sud ( $c f$. Béal et Odiot, infra, p. 95-96). Ces sites semblent se développer de façon privilégiée dans des secteurs à lit unique, si tant est que notre image du cours du fleuve soit pertinente.

\section{LES VOIES TERRESTRES}

\section{LES VOIES EST-OUEST}

Une analyse régressive à partir de documents planimétriques (cartes d'état-major du XIX $\mathrm{X}^{\mathrm{c}}$ s., cartes IGN au $1 / 20000$, tableau d'assemblage du cadastre napoléo- 
nien) a permis de mettre en évidence un certain nombre de chemins qui ne semblent pas participer à la logique d'occupation médiévale et peuvent refléter une réalité plus ancienne (Chouquer, Jung, 1996). Ces voies évitent en effet les centres de peuplements médiévaux et lorsque l'on possède une carte archéologique fournie, comme c'est le cas pour la plaine du Tricastin, on remarque qu'elles sont fréquemment jalonnées par des sites antiques. Un certain nombre de ces chemins partent du Rhône pour rejoindre des agglomérations antiques, la voie d'Agrippa ou l'arrière-pays tricastin. On présentera ici ceux qui sont les mieux documentés.

Dans la plaine valentinoise, différents chemins traversent la plaine en venant de la vallée de l'Isère et de celle de la Drôme pour converger vers le site de La Motte, au sud de Valence face à Soyons (fig. 34, itinéraires $\mathrm{n}^{\text {os }} 1$ et 2).

La vallée de la Drôme est empruntée par l'itinéraire des Alpes cottiennes attesté dès la Protohistoire et également connu par les itinéraires antiques (fig. $34, n^{\circ} 3$ ). Il s'agit de la voic joignant Valence à Die, Luc-en-Diois et Gap. Huit bornes milliaires datées des III et IV $^{\mathrm{e}}$ s. ont été retrouvées le long de cet itinéraire dans la vallée de la Drôme, entre Saillans et Pontaix (Desaye, 1989).

En Valdaine, le chemin Gontardin (fig. 34, $n^{\circ} 4$ ) traverse le bassin de part en part pour relier Montélimar à la vallée de la Drôme. Il est jalonné par de nombreux sites antiques. Un autre chemin (fig. $34, n^{\circ} 5$ ), mis en évidence par des analyses archéomorphologiques, est attesté par des sondages archéologiques lors du suivi des tranchées d'irrigation en Valdaine par J.-F. Berger (1996a) et lors de la phase de sondages de l'opération TGV-Méditerranée (J.-M. Treffort). Il relie la vallée du Rhône, à la hauteur de la commune des Tourrettes, à la haute vallée du Jabron en direction de Dieulefit, probable agglomération antique.

Au niveau de la plaine du Tricastin, l'agglomération antique de Bourg-Saint-Andéol est reliée à la cité de Saint-Paul-Trois-Châteaux par les $\mathrm{X}^{\mathrm{c}}, \mathrm{IX}^{\mathrm{c}}$ et $\mathrm{VIII}^{\mathrm{e}}$ decumani du cadastre $\mathrm{B}$ d'Orange (fig. $34, \mathrm{n}^{\circ} 6$ ). Ils forment un itinéraire qui traverse la plaine en dessinant une série de baïonnettes. Cet itinéraire a été mis en évidence à la sortie de Saint-Paul-Trois-Châteaux lors de la fouille par V. Bel de la nécropole du Valladas (Bel, Benoît, 1986).

Par ailleurs Bourg-Saint-Andéol (Bergoïata) est relié à la vallée de la Berre et à la mutatio de Novemcraris par un chemin jalonné de nombreux sites antiques (fig. 34, $\left.\mathrm{n}^{\circ} 7\right)$.

Un autre itinéraire coupant la plaine en diagonale relie l'oppidum de Saint-Étienne-de-Dion en Ardèche à celui du Moulon dans la Drôme (fig. $34, \mathrm{n}^{\circ} 8$ ).

Tous ces chemins reliant les sites de la rive ardéchoise à ceux de la rive drômoise traversent le Rhône à l'emplacement ou à proximité d'un haut-fond du fleuve où l'on peut envisager un passage à gué ou l'ancrage d'un bac.

\section{LES VOIES NORD-SUD}

On peut citer trois grands itinéraires routiers concurrençant la voie fluviale. Seule la voie d'Agrippa est connue par les itinéraires antiques. Les autres voies sont des reconstitutions à partir de la découverte de milliaires ou des études de carto-interprétation.

\section{LA VOIE D'AGRIPPA}

La voie d'Agrippa constitue la voie terrestre nord-sud principale. Entre Valence et Orange, elle reprend probablement le tracé d'un itinéraire plus ancien évoqué par Strabon (IV, I, 2) qui passait par Arausio, Aeria (oppidum de Barry ?) et Valentia. Le tracé de la voie d'Agrippa est connu dans ses grandes lignes par les travaux de Florian Vallentin (1880) qui l'a reconstitué après avoir recensé les milliaires trouvés le long de la voie et identifié les mutationes citées par les itinéraires antiques. La Table de Peutinger, l'Itinéraire Hierosolomytain et l'Anonyme de Ravenne mentionnent les relais jalonnant la route. Entre Valence et Orange ils indiquent quatre mutationes : Umbenno (Les Battendons, commune d'Étoile-surRhône), Bantiana (Banse, commune de Saulce-surRhône), Novemcraris (Le Logis-de-Berre, commune des Granges-Gontardes), Senomagos/Ad Letoce (Saint-Pierre de Sénos, commune de Bollène; Bollène) et une mansio: Acunum (Montélimar). Neuf milliaires ont été retrouvés le long de la voie entre Valence et Orange, le plus ancien est dédié à Tibère (CIL XVII, 165), les autres concernent des empereurs du Bas-Empire (Aurélien [CIL XVII, 160, 164], Constantin [CIL XVII, 163, 166], Antonin le Pieux [CIL XVII, 161], Dalmatius [CIL XVII, 163a]).

Entre Montélimar et Orange, la traversée des affluents du Rhône s'effectue juste en amont des cônes de déjections de ces rivières (Roubion/Jabron, Berre, 
Lez, Lauzon, Aygues), alors qu'elles présentent encore un cours unique et relativement stable. Leur traversée devait s'effectuer à gué ${ }^{44}$ durant les périodes d'étiage et par bac lors des hautes eaux, et pour l'instant aucun pont n'est attesté. Il faut aussi remarquer que les relais cités par les itinéraires antiques sont fréquemment situés aux franchissements de ces cours d'eaux (Acunum sur le Roubion/Jabron, Novemcraris sur la Berre, Ad Letoce sur le Lez.).

L'analyse archéomorphologique du réseau routier et la présence de sites antiques importants permettent de mettre en évidence la présence de dédoublements de la voie d'Agrippa. Entre Montélimar et Le Logis-de-Berre, une voie secondaire passe par le site du Palais à Châtcauneuf-du-Rhône (Jung, 1994), par les massifs du défilé de Donzère et l'agglomération de Donzère, permettant un débouché sur le Rhône. Plus au sud, un itinéraire semble éviter le massif d'Uchaux entre Bollène et Orange et longer la vallée du Rhône par Mondragon et Mornas, avant de rejoindre Orange.

Les rares observations archéologiques concernant la voie d'Agrippa montrent que sa matérialisation varie en fonction des tronçons étudiés. Des sondages ont été effectués sur le tronçon nommé La Levade entre SaintPierre de Sénos et Bollène (Vaucluse) par P. Faucher en 1882 (Rochetin, 1883). Ce tronçon entre les rivières du Lauzon et du Lez se présente en surélévation par rapport au niveau du terrain naturel souvent inondé. Les observations effectuées lors des sondages montrent que la voie, large de $5 \mathrm{~m}$, est construite de gravillons. Le même type de matériau est employé pour le tronçon entre les massifs d'Uchaux et la rivière de l'Eygues, au nord d'Orange (Rochetin, 1883). Au Logis-de-Berre, la voie, qui traverse l'agglomération, est constituée par des couches de galets, de graviers liés au mortier et de scories (Boisse, 1968). Elle présente une largeur de près de $6 \mathrm{~m}$. Sur les hautes terrasses rissiennes du Rhône, qui surplombent Le Logisde-Berre, les fossés de la route bien visibles sur les photographies aériennes devaient être la seule matérialisation au sol de la voie, la bande de roulement étant constituée par les graviers affleurants de la terrasse (sondages TGV-Méditerranée dirigés par C. Ronco).

La construction de la route n'est donc pas uniforme. Elle est véritablement construite dans les zones humides

44. Au Logis de Berre, la mention de "Pas de la Berre " évoque un passage à gué. ou inondables, ou à proximité et à l'intérieur des agglomérations qu'elle traverse. Sur les affleurements des terrasses rhodaniennes et les plateaux, elle correspond à un chemin de terre éventuellement encadré par des fossés. Cette constatation ne concerne pas uniquement la voie d'Agrippa, mais l'ensemble des routes que l'on a pu recouper lors des sondages du TGV-Méditerranée. P. Sillières (1990, p. 566-573 et 651), entre autres, souligne également cette hétérogénéité dans la construction des voies romaines.

La voie d'Agrippa ne structure aucunement l'habitat dispersé antique. En revanche, les calculs statistiques effectués à partir de la base des sites montrent l'importance du réseau routier dans l'implantation et la perduration des sites antiques. En Tricastin, les plus importants d'entre eux sont implantés à proximité de carrefours de voies qui sont constitués par des axes présentant un intérêt régional et des axes ayant un rôle dans la desserte locale (axes du cadastre B d'Orange). La voie dessert des agglomérations d'importance variée, des chefs-lieux de cités (Valence, Orange), ou des agglomérations secondaires comme Le Logis-de-Berre (Novemcraris : agglomération dès le Premier Âge du Fer et mutatio) qui semble structurer autour de lui un certain nombre de sites antiques et sert de rclais dans l'ćconomic locale ou des cités.

\section{LA VOIE PROTOHISTORIQUE DE VIENNE À MARSEILLE}

Une voie, probablement protohistorique (Chapotat, 1981), à l'écart du fleuve, emprunte l'arrière-pays drômois en passant par Romans-sur-Isère, Chabeuil, Crest où elle rejoint la voie des Alpes (Valence, Die, Luc-en-Diois, Gap). Cette première partie, peu accidentée, est encore largement reprise par une route départementale. Le profil de la voie entre Crest, Dieulefit, Le Pègue et Vaison-laRomaine est plus irrégulier avec le passage de cols. Si les grandes lignes du tracé proposé par G. Chapotat peuvent être retenues, certains tronçons paraissent peu probables, notamment entre Le Pègue et Vaison où l'auteur fait passer la piste par des reliefs très accidentés, évitant Nyons. La partie de la voie entre Vienne et la Drôme est encore utilisée au XVI ${ }^{e}$ s. Elle présente en effet l'avantage d'être plus courte, moins exposée aux avatars du fleuve et surtout être exempte de péage (Estienne, 1553). La voie qui pérennise le tracé de la voie d'Agrippa, appelée " grand chemin » au nord du Logis-de-Berre en 1701 
(Peloux, 1993) ou " chemin ferré " sur le cadastre napoléonien entre Le Logis-de-Berre et Bollène, et la route royale étaient effectivement jalonnées de nombreux péages ${ }^{45}$.

Pour l'Antiquité, l'importance de la voie décrite par G. Chapotat est difficilement appréciable. Il faut plus certainement envisager son rôle dans la desserte locale à régionale, avec des tronçons plus actifs que d'autres, plutôt que d'y voir un itinéraire suprarégional, qui structure les relations au niveau de la Province.

\section{La voie des Helviens}

Même si aucun itinéraire ne la mentionne, une voie nord-sud suivait la rive droite du Rhône. Les nombreux milliaires (CIL XVII, 1986) retrouvés à Cruas, Meysse, Rochemaure, Le Teil, Viviers et Bourg-Saint-Andéol l'attestent. Son tracé correspond dans ses grandes lignes à celui de la RN 86 qui borde le Rhône. Une bifurcation sur Alba s'effectuait au niveau du Teil (Napoli, Rebuffat, 1992) et un autre axe descendait depuis le chef-lieu de cité des Helviens en direction de Nîmes par Barjac et Uzès.

La largeur de la plaine et la densité de son occupation ont permis le développement d'un système de voiric particulièrement dense. Les axes est-ouest ou nord-sud, liés aux échanges économiques à grande et moyenne distance, sont renforcés par le réseau de communications offert par les chemins centuriés. Cette trame permettant des échanges denses et variés (choix de différents itinéraires en fonction des charges à transporter) montre combien il était possible pendant les périodes où le fleuve était difficilement navigable (variations saisonnières) de trouver des itinéraires de remplacement et de maintenir de façon constante un intense suivi des relations commerciales.

\section{VIVRE AU BORD DU FLEUVE}

D’une façon générale, la vision actuelle de la vallée du Rhône dans l'Antiquité tranche considérablement avec celle que les archéologues en avaient il y a une trentaine

45. Cf. la liste des péages par terre et également par eau de la Drôme en 1771 (Lacroix, 1902). d'années. C. Boisse (1968), entre autres, définissait cette plaine comme hostile et inondable. Cette perception s'explique facilement par les crues spectaculaires qui se sont succédé durant le XIX's. $\mathbf{s}(1840,1856 \ldots)$ et par les différentes zones palustres qui jalonnaient la plaine. Elle a été accentuée par la création dans les années cinquante d'une série de canaux de dérivation du fleuve, qui ont donné à la vallée son aspect actuel.

\section{UN HABITAT DENSE ET VARIÉ}

Les prospections systématiques ainsi que les observations de coupes, entreprises depuis une quinzaine d'années, ont montré la proximité de l'habitat antique avec le cours actuel du fleuve. Ainsi, dans le secteur de Pierrelatte, les sites de Sainte-Foy et du Pin ne sont distants de la rive actuelle du Rhône que de quelques centaines de mètres. Si quelques auteurs (Boisse, 1968; Boissier, 1979) ont évoqué la possibilité d'un passage du fleuve dans un chenal au centre de la plaine, les observations effectuées dans le cadre du TGV-Méditerranée ont permis de réfuter cette hypothèse et ont montré que, dans le secteur qu'il traverse, la majeure partie des signaturcs alluvialcs ćtait due aux apports détritiques des affluents du Rhône.

Les observations stratigraphiques sont rares à proximité du lit mineur du Rhône et, si quelques traces d'inondations du fleuve ont pu être observées, il faut désormais considérer que la majeure partie des recouvrements qui masquent les sites antiques est moderne.

Il s'agit désormais d'aborder la fouille d'édifices antiques en bordure du fleuve. On n'a pas pu observer, entre autres, si ces bâtiments étaient dotés, comme les édifices du XVIII ${ }^{\mathrm{e}} \mathrm{s}$., de systèmes de protection des crues (murs de clôtures avec portes étanches, etc.).

Néanmoins, la densité de l'occupation montre combien l'attrait du fleuve était plus important que les nuisances saisonnières.

Cette possibilité de vivre à proximité du fleuve est confirmée par la forma d'Orange. En cffet, à proximité du Rhône les tarifs de locations des terres sont les plus élevés, trois fois plus que la moyenne (Odiot, 1994; Berger et al., 1997). Cette variation des loyers montre bien l'importance que la colonie attachait à la tenure de ces terres qui se révèlent aussi parmi les meilleures de la plaine. Les quelques subsesciva attestées sont localisées 
dans des secteurs de forte turbulence du cours et peuvent correspondre à des marges.

D'autre part, à proximité immédiate du fleuve, les mentions « remissa in aquam " et " nova aera » montrent à la fois des variations latérales du cours du fleuve et la lourdeur des travaux engagés pour l'assainissement des terres. À l'échelle de la centuriation, le décompte de ces terres nouvellement conquises ou abandonnées, tout comme la densité de l'habitat sur la façade occidentale de la plaine indiquent l'intérêt que suscitent ces terres exposées régulièrement au fleuve. L'image des établissements ruraux connus par prospections aériennes ou par les rares observations de fouilles ne montre pas de discrimination dans le type d'habitat. Grosses villae et "petits" sites sont attirés de la même façon par le fleuve.

Malgré les prospections aucune digue ni aucune levée de protection des crues n'ont été mises en évidence, mais l'importance des travaux modernes de « canalisation du fleuve " ou les recouvrements sédimentaires ont pu masquer de tels aménagements.

\section{UNE MAÎTRISE PROGRESSIVE DES RIVES ?}

Les prospections pédestres systématiques (Odiot, 1994) ont montré que, dans le secteur situé à l'ouest du cardo maximus et au nord du decumanus maximus, les implantations s'effectuaient progressivement du sud vers le nord, de la période augustéenne à la période flavienne. Cette constatation peut, en partie, s'expliquer par des contraintes physiques et hydrographiques. En effet, dans la partie nord de la plaine du Tricastin, le débouché du défilé de Donzère et le confluent Rhône/Berre constituent une zone de grande instabilité dont on peut penser que la maîtrise des terres (sans doute impossible) a été toutefois facilitée par l'endiguement de la Berre et la mise en place du réseau de drainage ( $c$. infra). En revanche, à la hauteur du $\mathrm{X}^{\mathrm{e}}$ decumanus face à Bourg-Saint-Andéol, une multitude de facteurs, comme la nécessité de franchir le flcuve pour les échanges commerciaux avec le territoire helvien, la possibilité d'écouler ses produits par la voie fluviale et la bonification des terres drainées par le $\mathrm{X}^{\mathrm{e}}$ decumanus ( $c f$. infra), sont autant d'éléments justifiant l'implantation prioritaire des colons et l'attirance des locataires des terres pour ce secteur.

\section{DRAINAGE, ENDIGUEMENT DES AFFLUENTS ET ABAISSEMENT DE LA NAPPE PHRÉATIQUE}

Les importants travaux de drainage dans la plaine du Tricastin sont liés tant à la mise en culture des terres qu'à l'installation des habitats. Les multiples observations archéologiques, effectuées sur les fossés de drainage lors des travaux du TGV-Méditerranée (Berger, Jung, 1996), montrent l'importance de ces structures dans la gestion des eaux météoriques et dans la régulation des nappes phréatiques. La plupart des axes centuriaux et des limites internes à la centurie sont matérialisés par un fossé ou une voie bordée de fossés qui accélèrent l'écoulement des eaux de surface. Sur certains sites où un ensemble de. fossés a pu être étudié, l'arborescence du réseau et sa hiérarchisation ont été appréhendées. Ainsi, à Mondragon "Le Duc » (sondages TGV-Méditerranée G. Alfonso) ou Mondragon "Les Brassières " (fouille TGV-Méditerranée C. Vermeulen), les fossés de drainage secondaires se déversent dans un collecteur principal (correspondant ou non à une limite de centurie), qui conduit les eaux vers le Lez ou vers le Rhône. Plus au nord, le $\mathrm{X}^{\mathrm{e}}$ decumanus qui traverse la plaine de Pierrelatte a dû jouer ce même rôle de collecteur principal. Dans le secteur de la Berre, la route antique menant de Novemcraris à Augusta Tricastinorum est bordée de puissants fossés qui reçoivent les eaux provenant des collines de La Garde-Adhémar et les évacuent dans des decumani du cadastre B d'Orange (Jung, 1997). Les cours d'eau sont également régulés par des travaux d'endiguement. Les marbres d'Orange représentent un cours de la Berre rectiligne à la sortie de sa vallée, au niveau de Novemcraris, qui contraste avec celui représenté en aval où la Berre dessine une série de méandres. Cette représentation rectiligne indique probablement une rectification ou un endiguement à cet endroit précis. L'ensemble de ces aménagements de surface induit une baisse des nappes phréatiques de surface, largement appauvries et régulées. Les terrains ainsi drainés sont bonifiés. Ce travail de mise en valeur des terres semble s'échelonner dans le temps (Odiot, 1994) accompagnant une progression des implantations de sites du sud vers le nord. De plus, dans certains secteurs, on observe un véritable nivellement de la plaine. Ainsi près de Pierrelatte à L'Espitalet (Berger $e t$ al., 1997 ; Brochier, 1997), une série de dépressions (correspondant à des paléochenaux du Rhône) est volontairement comblée durant le Haut-Empire. 


\section{UN DENSE RÉSEAU DE COMMUNICATIONS}

La mise en valeur des terres de la plaine, jusqu'aux rives du fleuve, semble directement liée à l'effort colonial et au développement d'une agriculture extensive. Depuis la découverte de la villa du Molard (Dechandol, 1982 ; Odiot, 1997), les témoignages d'une viticulture très active dans le Tricastin s'accumulent, qu'il s'agissent de nouveaux établissements viticoles ou de traces de plantations de vignes (Séris, 1996 ; Roger, 1999). Les décapages ou les sondages liés aux travaux du TGV-Méditerranée (Boissinot, 1997) tout comme les photographies aériennes (prospections aériennes Odiot) montrent l'importance des superficies consacrées aux vignobles. Cette production de masse semble trouver un débouché à la fois vers le Rhône et vers la voie d'Agrippa, comme le montre l'itinéraire doublant la voie d'Agrippa, entre Le Logis-de-Berre et Montélimar, et reliant la villa du Molard soit à la mutatio de Novemcraris, soit à l'agglomération antique de Donzère et au Rhône. L'établissement viticole du Fayn (Séris, 1996), à proximité de Novemcraris, s'insère dans un réseau de chemins qui lui donnent accès à la voie d'Agrippa et à la mutatio. À cette production, qui scmblc cn partic tournćc vers l'cxportation, on pcut ajouter celle de la pierre du Midi avec les carrières d'Augusta Tricastinorum dont l'exportation vers le nord est probable (Odiot et al., 1992).

La moyenne vallée du Rhône a donc, durant l'Antiquité, été l'objet d'une intense mise en valeur, tant dans le cadre de l'établissement des voies stratégiques à l'échelle de l'Empire ou du niveau local qu'au niveau de la régulation du milieu par le réseau cadastral. Les cités et le maillage des agglomérations et des exploitations rurales sont en effet desservis par les "limites" du réseau centurié et les chemins locaux, mais aussi par l'ensemble de la voirie de grande circulation. L'aménagement global de la moyenne vallée du Rhône a pris la mesure du fleuve et de ses variations en essayant, au prix de travaux de grande ampleur, de répondre au mieux aux besoins des colonies. Le réseau centurié semble avoir particulièrement bien fonctionné dans la plaine du Tricastin et avoir été adapté en fonction de l'ensemble des réalités physiques et hydrographiques. Instrument administratif pour l'imposition, mais aussi moyen de communication interne, il apparaît surtout particulièrement performant comme outil de drainage des eaux météoriques, de régulation des nappes phréatiques et des affluents du Rhône. La pertinence de cette infrastructure est telle que son maillage restera utilisé par l'ensemble des communautés agricoles qui exploiteront la plaine après la période romaine. Ainsi de nombreux fossés de drainage continueront à être entretenus parfois jusqu'à nos jours (Berger, Jung, 1996 ; Jung, 1997).

\section{Remerciements}

Nous tenons à remercier plus particulièrement Jean-François Berger, Jacques-Léopold Brochier, François Favory, Philippe Leveau et François Vaireaux pour leurs conseils et leurs informations sur le Rhône, ainsi que tous nos collègues archéologues avec lesquels nous avons collaboré sur le terrain. 